TOKYO J. MATH.

VOL. 6, No. 2, 1983

\title{
Homeomorphisms with the Pseudo Orbit Tracing Property of the Cantor Set
}

\author{
Masahito DATEYAMA \\ Tokyo Metropolitan University \\ (Communicated by M. Iwano)
}

Let $X$ be a compact metric space with metric $d$, and $f$ be a homeomorphism from $X$ onto itself. A sequence $\left\{x_{i}\right\}_{i=-\infty}^{\infty}$ is said to be a $\delta$-pseudoorbit of $f$ if $d\left(f x_{i}, x_{i+1}\right)<\delta$ holds for all $i \in Z$. $(X, f)$ is said to have the pseudo orbit tracing property (abbrev. P.O.T.P.) if for every $\varepsilon>0$ there is $\delta>0$ such that, for every $\delta$-pseudo-orbit $\left\{x_{i}\right\}_{i=-\infty}^{\infty} \subset X$, there exists an $x \in X$ such that $d\left(f^{i} x, x_{i}\right)<\varepsilon$ for all $i \in Z$. Let $C \subset[0,1]$ be the Cantor set: i.e. $C$ is the set of the numbers $x \in[0,1]$ with $x=3^{-1} a_{1}+3^{-2} a_{2}+\cdots$ $\left(a_{i}=0\right.$ or 2 for $i \geqq 1$ ). We denote by $\mathscr{H}(C)$ the set of all homeomorphisms on $C$, and by $\mathscr{P}(C)$ the set of all homeomorphisms with the P.O.T.P.. Define the metric $\bar{d}$ on $\mathscr{H}(C)$ by $\bar{d}(f, g)=\max _{x \in C} d(f x, g x), f, g \in \mathscr{H}(C)$. Then $\mathscr{H}(C)$ is a Banach space.

In this paper we prove:

TheOREM. $\mathscr{P}(C)$ is dense in $\mathscr{H}(C)$.

For $r \geqq 1$, we call the set $C \cap\left[3^{-r} i, 3^{-r}(i+1)\right]\left(0 \leqq i \leqq 3^{r}-1\right)$ a Cantor subinterval with rank $r$ if $C \cap\left(3^{-r} i, 3^{-r}(i+1)\right) \neq \varnothing$. We denote by $I(i, r)$, the $i$-th Cantor subinterval with the rank $r$ from the left. Clearly $C=\bigcup_{i=1}^{2 r} I(i, r)$ and $I(i, r)=I(2 i-1, r+1) \cup I(2 i, r+1)$. We call $g \in \mathscr{H}(C)$ a generalized permutation if there exists $r \geqq 1$ such that the following i) and ii) hold:

i) For every $1 \leqq i \leqq 2^{r}$, there exist $s=s(i) \geqq 1$ and $1 \leqq j=j(i) \leqq 2^{*}$ such that $g(I(i, r))=I(j, s)$, and

ii) For every $1 \leqq i \leqq 2^{r}$, there exists $k=k(i) \in R$ such that $g(x)=$ $3^{r-s(i)} x+k, x \in I(i, r)$.

Denote by $\mathscr{G}$ the set of all generalized permutations. Then $\mathscr{G}$ is dense in $\mathscr{H}(C)$. In fact, take $f \in \mathscr{H}(C)$ and $r \geqq 1$. Choose $s \geqq 1$ such that $d(x, y)<3^{-s}$ implies $d(f x, f y)<3^{-r}$. Then for every $1 \leqq i \leqq 2^{s}$ there exists 
$1 \leqq i_{j} \leqq 2^{r}$ such that $f(I(j, s)) \subset I\left(i_{j}, r\right)$. Since $f$ is onto, for every $1 \leqq i \leqq 2^{r}$ there exist $n \geqq 1$ and $j_{1}, \cdots, j_{n}>0$ such that $f\left(\bigcup_{k=1}^{n} I\left(j_{k}, s\right)\right)=I(i, r)$. Since $I(i, r)$ is a disjoint union of $n$ Cantor subintervals (say $I(i, r)=\bigcup_{k=1}^{n} I\left(i_{k}, r_{k}\right)$ ), we can construct $g \in \mathscr{G}$ with $g\left(I\left(j_{k}, s\right)\right)=I\left(i_{k}, r_{k}\right), 1 \leqq k \leqq n$. It is easy to check that $\bar{d}(f, g)<3^{-r}$. Since $r$ is arbitrary, our requirement is obtained.

Let $S$ be the finite set with the discrete topology, and $\Sigma=S^{z}$ be the bilateral infinite product space with metric $d^{\prime}$ defined by

$$
d^{\prime}(s, t)=\max _{i \in Z} \delta\left(s_{i}, t_{i}\right) / 2^{|i|} \quad\left(s=\left(s_{i}\right), t=\left(t_{i}\right) \in \Sigma\right)
$$

where $\delta\left(s_{i}, t_{i}\right)=1$ if $s_{i} \neq t_{i}$, and $=0$ if $s_{i}=t_{i}$. Define the shift homeomorphism $\sigma$ of $\Sigma$ by $(\sigma(s))_{i}=s_{i+1}, i \in Z$. If $X$ is a closed subset of $\Sigma$ with $\sigma X=X$, then $(X, \sigma)$ is called a subshift. A subshift $(X, \sigma)$ is said to be of finite type if there exist $L>0$ and $B \subset S^{L}$ such that $X=\left\{s=\left(s_{i}\right) \in \Sigma\right.$ : $\left(s_{i}, \cdots, s_{i+L-1}\right) \in B$ for all $\left.i \in Z\right\} . \quad L$ is called the order of $(X, \sigma)$. It is proved in P. Walters [5] that a subshift $(X, \sigma)$ has the P.O.T.P. iff $(X, \sigma)$ is of finite type.

Proof of Theorem. Since $\mathscr{G}$ is dense in $\mathscr{H}(C)$, it is enough to prove that every $g \in \mathscr{G}$ has the P.O.T.P.. Take $g \in \mathscr{G}$, then there exists $r_{0} \geqq 1$ such that for every $r \geqq r_{0}, g$ satisfies i) and ii) in the definition of generalized permutation. Let $\varepsilon>0$, choose $r \geqq r_{0}$ such that $3^{-r}<\varepsilon$. For every $x \in C$ and every $i \in Z$, define $x_{i} \in\left\{1, \cdots, 2^{r}\right\}$ by $g^{i}(x) \in I\left(x_{i}, r\right)$. By the definition of generalized permutation, it follows that $\bigcap_{j=1}^{n} g^{j}\left(I\left(x_{-j}, r\right)\right)$ $(n \geqq 1)$ are Cantor subintervals for all $x \in C$. Put $\Sigma=F^{z}$ where $F=$ $\left\{1, \cdots, 2^{r}\right\}$ and let $\sigma$ be the shift homeomorphism of $\Sigma$. For $x \in X$, define $h(x)=\left(x_{i}\right)$. Then $h: C \rightarrow \Sigma$ is a continuous map and $h \circ g=\sigma \circ h$ holds. Let us put

$$
A=\left\{x \in C: \bigcap_{j=1}^{\infty} g^{j}\left(x_{-j}, r\right) \varsubsetneqq I\left(x_{0}, r\right)\right\} .
$$

For $x \in A$, denote by $n(x)$ the minimum number such that

$$
\bigcap_{j=1}^{n(x)} g^{j}\left(I\left(x_{-j}, r\right)\right) \varsubsetneqq I\left(x_{0}, r\right),
$$

and for $x \in C \backslash A$, denote by $n(x)$ the minimum number such that

$$
\bigcap_{j=1}^{n(x)} g^{j}\left(I\left(x_{-j}, r\right)\right)=\bigcap_{j=1}^{\infty} g^{j}\left(I\left(x_{-j}, r\right)\right) \supset I\left(x_{0}, r\right) .
$$

Then we have

$$
\max _{x \in C} \operatorname{rank} \bigcap_{j=1}^{n(x)} g^{j}\left(I\left(x_{-j}, r\right)\right)=\max _{i \in F} \operatorname{rank} g(I(i, r))<\infty,
$$

so that $\left\{\bigcap_{j=1}^{n(x)} g^{j}\left(I\left(x_{-j}, r\right)\right): x \in C\right\}$ is finite. If $\bigcap_{j=1}^{n(x)} g^{j}\left(I\left(x_{-j}, r\right)\right)=\bigcap_{j=1}^{n\left(x^{\prime}\right)} g^{j}\left(I\left(x_{-j}^{\prime}\right.\right.$, 
$r))\left(x, x^{\prime} \in C\right)$, then we get $x_{-j}=x_{-j}^{\prime}\left(1 \leqq j \leqq \min \left(n(x), n\left(x^{\prime}\right)\right)\right)$. Since $n(x)$ and $n\left(x^{\prime}\right)$ are minimal, $n(x)=n\left(x^{\prime}\right)$ holds. This implies that $\{n(x): x \in C\}$ is finite. Put $N=\max _{x \in C} n(x)$ and

$$
B=\left\{\left(i_{0}, i_{-1}, \cdots, i_{-N}\right) \in F^{N+1}: \bigcap_{j=0}^{N} g^{j}\left(I\left(i_{-j}, r\right)\right) \neq \varnothing\right\} .
$$

Then $(h(C), \sigma)$ is a subshift of finite type of order $N+1$. To see this, set

$$
\Sigma_{B}=\left\{\left(i_{j}\right) \in \Sigma:\left(i_{j}, i_{j-1}, \cdots, i_{j-N}\right) \in B \text { for all } j \in \mathbb{Z}\right\} .
$$

Clearly $\sigma \Sigma_{B}=\Sigma_{B},\left(\Sigma_{B}, \sigma\right)$ is of finite type, and $h(C) \subset \Sigma_{B}$. To prove that $h(C) \supset \Sigma_{B}$, it is enough to show that

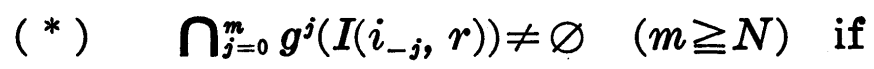

$$
\left(i_{-j}, i_{-(j+1)}, \cdots, i_{-(j+N)}\right) \in B \text { for all } 0 \leqq j \leqq m-N .
$$

We use induction with respect to $m$. When $m=N,\left({ }^{*}\right)$ is true. Assume that $\left(^{*}\right)$ holds for $m$. Take $\left(i_{-j}\right)_{j=0}^{m+1} \in F^{m+1}$ with $\left(i_{-j}, i_{-(j+1)}, \cdots, i_{-(j+N)}\right) \in B$ for all $0 \leqq j \leqq(m+1)-N$. By assumption we have $\bigcap_{j=1}^{m+1} g^{j}\left(I\left(i_{-j}, r\right)\right) \neq \varnothing$, and so $\bigcap_{j=0}^{N} g^{j}\left(I\left(i_{-j}, r\right)\right) \neq \varnothing$. Take $x \in \bigcap_{j=0}^{N} g^{j}\left(I\left(i_{-j}, r\right)\right)$. If $x \in A$, then $\bigcap_{j=1}^{N} g^{j}\left(I\left(i_{-j}, r\right)\right) \varsubsetneqq I\left(i_{0}, r\right)$. Therefore $\bigcap_{j=0}^{m+1} g^{j}\left(I\left(i_{-j}, r\right)\right)=\bigcap_{j=1}^{m+1} g^{j}\left(I\left(i_{-j}, r\right)\right) \neq \varnothing$. When $x \notin A, \bigcap_{j=1}^{m+1} g^{j}\left(I\left(i_{-j}, r\right)\right)=\bigcap_{j=1}^{N} g^{j}\left(I\left(i_{-j}, r\right)\right) \supset I\left(i_{0}, r\right)$, and so $\bigcap_{j=0}^{m+1} g^{j}\left(I\left(i_{-j}\right.\right.$, $r)) \supset I\left(i_{0}, r\right) \neq \varnothing$. Thus $(h(C), \sigma)$ is of finite type of order $N+1$. As before let $d^{\prime}$ be the metric of $\Sigma$ and $\varepsilon^{\prime}$ be a number such that $d^{\prime}(s, t)<\varepsilon^{\prime}\left(s=\left(s_{i}\right)\right.$, $\left.t=\left(t_{i}\right) \in \Sigma\right)$ implies $s_{0}=t_{0}$. Since $(h(C), \sigma)$ is of finite type, there exists $\delta>0$ such that for every $\delta$-pseudo-orbit $\left\{s^{n}\right\} \subset h(C)$, there is $s \in h(C)$ such that $d^{\prime}\left(\sigma^{n} s, s^{n}\right)<\varepsilon^{\prime}$. Choose $\eta>0$ such that $d(x, y)<\eta(x, y \in C)$ implies $d^{\prime}(h(x), h(y))<\delta$ and take an $\eta$-pseudo-orbit $\left\{x^{n}\right\} \subset C$ of $g$. Then $\left\{h\left(x^{n}\right)\right\}$ is a $\delta$-pseudo-orbit of $\sigma$. Hence there exists $x \in C$ such that $d^{\prime}\left(\sigma^{n} h(x)\right.$, $\left.h\left(x^{n}\right)\right)<\varepsilon^{\prime}$. This shows that $h\left(g^{n} x\right)_{0}=h\left(x^{n}\right)_{0}(n \in \boldsymbol{Z})$, and so $d\left(g^{n} x, x^{n}\right)<3^{-r}<\varepsilon$ $(n \in Z)$. The proof of the theorem is completed.

\section{References}

[1] N. Aokr, The splitting of zero-dimensional automorphisms and its application, to appear in Colloq. Math..

[2] R. BowEN, $\omega$-limit set for Axiom A diffeomorphisms, J. Differential Equations, 18 (1975), 333-339.

[3] M. Denker, C. Grillenderger and K. Sigmund, Ergodic Theory on Compact Spaces, Lecture Notes in Math., 527, Springer-Verlag, Berlin-Heidelberg-New York, 1976.

[4] M. Sears, Expansive self-homeomorphisms of the Cantor set, Math. Systems Theory, 6 (1972), 129-132.

[5] P. WALTERS, On the pseudo orbit tracing property and its relationship to stability, The 
Structure of Attractor in Dynamical Systems, Lecture Notes in Math., 668, SpringerVelag, Berlin-Heidelberg-New York, (1978), 231-244.

Present Address:

Diparturent OF Mathematics Osara City Univirgity SUGIMoto, SUMTYOsHI-KU OSARA, 558 